\title{
Get it strained
}

Stretching a sheet of graphene could induce a superconducting state. Similar strain-induced superconductivity may be realized at the interface between a topological crystalline insulator and a trivial band insulator.

\author{
Fakher F. Assaad
}

$\mathrm{O}$ ne of the reasons for the excitement surrounding graphene is that it represents a solid-state realization of the Dirac equation - the cornerstone of relativistic quantum theory unifying quantum mechanics and the special theory of relativity. The equation provides a mathematical framework for the relativistic description of the electron, and automatically accounts for the electron's spin and that of its antimatter partner, the positron. Stretching a sheet of graphene corresponds to the inclusion of a magnetic field in the equation ${ }^{1}$, as the strain applied to a two-dimensional honeycomb lattice of carbon atoms induces a 'pseudo-magnetic' field. Now, Evelyn Tang and Liang Fu report in Nature Physics that stretching graphene can, in essence, also produce a superconducting state ${ }^{2}$.

For low energies, the electronic structure of a sheet of graphene features linear dispersions known as Dirac cones. The vector potential associated with the strain-induced pseudo-magnetic field has opposite signs for graphene's two types of Dirac cone, which reflects the fact that elastic deformations, unlike real magnetic fields, do not violate time-reversal symmetry. For mathematical reasons, which are collectively known as index theorems, this effective pseudo-magnetic field leads to the formation of zero-energy modes ${ }^{3}$. The number of zero-energy modes corresponds to the number of pseudo-magnetic flux quanta threading the graphene sheet. Therefore, one obtains a flat band pinned at zero energy that is half-filled with electrons. The resulting large density of states at zero energy has been observed experimentally for so-called molecular graphene $e^{4}$ - carbon monoxide molecules carefully placed on a copper substrate in a hexagonal arrangement.

A partially filled flat band is generically very susceptible to electronic correlations. When the system becomes very large, at a constant pseudo-magnetic field the density of states at zero energy grows proportionally to the volume of the system. In this situation even weak correlations can play an important role by opening up a gap in the density of states at zero energy.
Occupied states are therefore shifted to lower energy leading to an energy gain. In other words, when the system becomes infinitely large - in the thermodynamic limit - it is bound to be unstable to a correlation-dominated collective state. Tang and Fu demonstrated that, assuming the electron-phonon coupling is the dominant interaction, one can expect that the stretching of a sheet of graphene will induce a superconducting state ${ }^{2}$. In contrast, dominant repulsive interactions have the potential to generate magnetic instabilities ${ }^{5,6}$.

Tang and $\mathrm{Fu}$ suggested how one could take this thought experiment into the laboratory ${ }^{2}$. The scheme they proposed is a self-organized realization of the straininduced superconductivity effect involving the physics of topological crystalline insulators. These materials are insulating in the bulk, but metallic at the surface. Their metallic surface state is robust due to crystal symmetries and not due to time-reversal symmetry, as is the case for ordinary topological insulators. This has important consequences and renders the metallic surface state of the topological crystalline insulator very similar to that of graphene, giving rise to the desired Dirac Hamiltonian.

But what about strain? To produce a uniform pseudo-magnetic field in the thermodynamic limit, one would need infinite strain, which would rip apart any crystalline structure. To get around this issue, the authors considered an interface between a topological crystalline insulator and a trivial band insulator (a semiconductor with no topological properties). The metallic surface state resides at the interface of the topological crystalline insulator and the trivial band insulator because the band insulator can essentially be considered a vacuum. By choosing a trivial band insulator with a different lattice constant than that of the topological crystalline insulator, a periodic array of dislocations, or a periodic strain, can be induced at the interface. This leads to the desired flat band. In semiconductors, the dominant correlation effects stem from the electron-phonon coupling such that an emergent superconducting state at the interface of the topological crystalline insulator and the trivial band insulator is expected. Note that graphene and topological crystalline insulator surface states differ from the topological insulator surface state. In the latter case, the Dirac point is pinned at time-reversalinvariant momenta, so it cannot meander in momentum space and the proposed mechanism of a strained-induced flat band fails?

Superconductivity stemming from a flat band is interesting in its own right as it is not explained by the Bardeen-Cooper-Schrieffer theory of superconductivity ${ }^{8}$. The density of states at the Fermi level is infinite and the Fermi energy vanishes. This means that one cannot rely blindly on the Migdal theorem ${ }^{9}$, which provides a precise method for calculating the electron-phonon coupling and predicting superconducting transition temperatures.

Topological crystalline and trivial band insulators are semiconductors that, taken on their own, often do not show dominant correlation effects. Put together, however, a piece of correlation-dominated physics emerges at the interface. This mechanism provides an interesting way to understand the observed superconductivity in IV-VI semiconductor interfaces ${ }^{10}$, and it may lead to a novel route to generate correlationdominated and exotic states of matter in partially filled flat bands.

Fakher F. Assaad is at the Institut für Theoretische Physik und Astrophysik, Universität Würzburg, Am Hubland, D-97074 Würzburg, Germany. e-mail:assaad@physik.uni-wuerzburg.de

References

1. Castro Neto, A. H., Guinea, F., Peres, N. M. R., Novoselov, K. S. \& Geim, A. K. Rev. Mod. Phys. 81, 109-162 (2009).

2. Tang, E. \& Fu, L. Nature Phys. 10, 964-969 (2014)

3. Aharonov, Y. \& Casher, A. Phys. Rev. A 19, 2461-2462 (1979).

4. Gomes, K. K., Mar, W., Ko, W., Guinea, F. \& Manoharan, H. C. Nature 483, 306-310 (2012).

5. Herbut, I. F. Phys. Rev. B 78, 205433 (2008).

6. Roy, B., Assaad, F. F. \& Herbut, I. F. Phys. Rev. X 4, 021042 (2014).

7. Liu, Y. et al. Nature Phys. 10, 294-299 (2014).

8. Bardeen, J., Cooper, L. N. \& Schrieffer, J. R. Phys. Rev. 106, 162-164 (1957).

9. Migdal, A. JETP 34, 996-1001 (1958).

10. Fogel, N. Ya. et al. Phys. Rev. Lett. 86, 512-515 (2001).

Published online: 2 November 2014 